\title{
Analytic Solutions of a Second-Order Functional Differential Equation with a State Derivative Dependent Delay
}

\author{
Jiraphorn Somsuwan and Keaitsuda Maneeruk Nakprasit \\ Department of Mathematics, Faculty of Science, Khon Kaen University, Khon Kaen 40002, Thailand \\ Correspondence should be addressed to Keaitsuda Maneeruk Nakprasit; kmaneeruk@hotmail.com
}

Received 1 July 2015; Accepted 20 September 2015

Academic Editor: Bao Qin Li

Copyright ( 2015 J. Somsuwan and K. M. Nakprasit. This is an open access article distributed under the Creative Commons Attribution License, which permits unrestricted use, distribution, and reproduction in any medium, provided the original work is properly cited.

\begin{abstract}
We investigate an analytic solution of the second-order differential equation with a state derivative dependent delay of the form $x^{\prime \prime}(z)=x\left(p(z)+b x^{\prime}(z)\right)$. Considering a convergent power series $g(z)$ of an auxiliary equation $\gamma^{2} g^{\prime \prime}(\gamma z) g^{\prime}(z)=\left[g\left(\gamma^{2} z\right)-\right.$ $p(g(\gamma z))] \gamma g^{\prime}(\gamma z)\left(g^{\prime}(z)\right)^{2}+p^{\prime \prime}(g(z))\left(g^{\prime}(z)\right)^{3}+\gamma g^{\prime}(\gamma z) g^{\prime \prime}(z)$ with the relation $p(z)+b x^{\prime}(z)=g\left(\gamma g^{-1}(z)\right)$, we obtain an analytic solution $x(z)$. Furthermore, we characterize a polynomial solution when $p(z)$ is a polynomial.
\end{abstract}

\section{Introduction}

The functional differential equation,

$$
\begin{aligned}
& x^{(m)}(z)=f\left(z, x^{\left(m_{1}\right)}\left(z-\tau_{1}(z)\right), x^{\left(m_{2}\right)}\left(z-\tau_{2}(z)\right),\right. \\
& \left.\ldots, x^{\left(m_{k}\right)}\left(z-\tau_{k}(z)\right)\right),
\end{aligned}
$$

where all $m_{i} \geq 0, \tau_{i} \geq 0$, provides a mathematical model for a physical or biological system in which the rate of change of system is determined not only by its present state, but also by its history (see $[1,2])$. In recent years, many authors studied the existence and the uniqueness of an analytic solution of a variety of these equations. In 1984, Eder [3] classified solutions of the functional differential equation $x^{\prime}(z)=$ $x(x(z))$ by using the Banach fixed point theorem. Let $a$ and $b$ be nonzero complex constants and $p(z)$ a complex function. The first-order functional differential equation $x^{\prime}(z)=x(a z+$ $b x(z)), x^{\prime}(z)=x(p(z)+b x(z))$, and $x^{\prime}(z)=x\left(p(z)+b x^{\prime}(z)\right)$ has been studied by Si and Cheng [4], Qiu and Liu [5], and Zhang [6], respectively.

In 2001 [7], Si and Wang investigated the existence of analytic solution of the second-order functional differential equation:

$$
x^{\prime \prime}(z)=x(a z+b x(z)) .
$$

In 2009, Liu and $\mathrm{Li}$ [8] studied the equation

$$
x^{\prime \prime}(z)+c x^{\prime}(z)=x(a z+b x(z)) .
$$

Observe that (3) can be reduced to (2) by setting $c=0$.

Next, the equation

$$
x^{\prime \prime}(z)=x\left(a z+b x^{\prime}(z)\right)
$$

has been studied by Si and Wang [9].

In order to obtain analytic solutions of (4), they constructed a corresponding auxiliary equation with parameter $\gamma$. The existence of solutions of an auxiliary equation depends on the condition of a parameter $\gamma$, such as $\gamma$ is in the unit circle and $\gamma$ is a root of unity which satisfies the Diophantine condition.

In this paper, we study the existence of analytic solutions of the second-order differential equation with a state derivative dependent delay of the form

$$
x^{\prime \prime}(z)=x\left(p(z)+b x^{\prime}(z)\right) .
$$

If $p(z)=a z$, then (5) reduces to (4).

Note that in this paper, we will study three cases of parameter $\gamma$ in a corresponding auxiliary equation. One of them is the case that $\gamma$ is a root of unity satisfying the Brjuno condition. 
To construct an auxiliary equation, we set

$$
y(z)=p(z)+b x^{\prime}(z)
$$

Then

$$
x(z)=x\left(z_{0}\right)+\frac{1}{b} \int_{z_{0}}^{z}(y(s)-p(s)) d s
$$

where $z_{0}$ is a complex constant. In particular, we have

$$
x(y(z))=x\left(z_{0}\right)+\frac{1}{b} \int_{z_{0}}^{y(z)}(y(s)-p(s)) d s .
$$

Applying relations (6) and (8) to (5), we obtain

$$
\begin{aligned}
\frac{1}{b}\left(y^{\prime}(z)-p^{\prime}(z)\right)= & x\left(z_{0}\right) \\
& +\frac{1}{b} \int_{z_{0}}^{y(z)}(y(s)-p(s)) d s .
\end{aligned}
$$

We construct the corresponding equation by differentiating both sides of (9) with respect to $z$. This yields

$$
y^{\prime \prime}(z)-p^{\prime \prime}(z)=(y(y(z))-p(y(z))) y^{\prime}(z) .
$$

\section{Analytic Solutions of (10)}

Consider the auxiliary equation

$$
\begin{aligned}
\gamma^{2} g^{\prime \prime}(\gamma z) g^{\prime}(z) & \\
= & \left(g\left(\gamma^{2} z\right)-p(g(\gamma z))\right) \gamma g^{\prime}(\gamma z)\left(g^{\prime}(z)\right)^{2} \\
& +p^{\prime \prime}(g(z))\left(g^{\prime}(z)\right)^{3}+\gamma g^{\prime}(\gamma z) g^{\prime \prime}(z)
\end{aligned}
$$

with initial value conditions $g(0)=0$ and $g^{\prime}(0)=\eta \neq 0$, where $\gamma, \eta$ are complex numbers. Observe that if $g(z)$ is an analytic solution of (11), then (10) has an analytic solution of the from $y(z)=g\left(\gamma g^{-1}(z)\right)$. Equation (11) can be reduced equivalently to the integro-differential equation

$$
\begin{aligned}
& \gamma g^{\prime}(\gamma z)=g^{\prime}(z)\left(\gamma+\int_{0}^{z}\left(\gamma g^{\prime}(\gamma s) g\left(\gamma^{2} s\right)\right.\right. \\
& \left.\left.-\gamma g^{\prime}(\gamma s) p(g(\gamma s))+p^{\prime \prime}(g(s)) g^{\prime}(s)\right) d s\right),
\end{aligned}
$$

where $g(0)=0$ and $g^{\prime}(0)=\eta \neq 0$. To construct analytic solutions of (12), we separate our study on the conditions of the parameter $\gamma$ as follows:

(H1) $0<|\gamma|<1$;

(H2) $\gamma=e^{2 \pi i \theta}$, where $\theta \in \mathbb{R} \backslash \mathbb{Q}$ is a Brjuno number; that is, $B(\theta)=\sum_{k=0}^{\infty} \log q_{k+1} / q_{k}<\infty$, where $\left\{p_{k} / q_{k}\right\}$ denotes the sequence of partial fraction of the continued fraction expansion of $\theta$;
(H3) $\gamma=e^{2 \pi i q / p}$ for some integers $p \in \mathbb{N}$ with $p \geq 2$ and $q \in \mathbb{Z} \backslash\{0\}$, and $\gamma \neq e^{2 \pi i l / k}$ for all $1 \leq k \leq p-1$ and $l \in \mathbb{Z} \backslash\{0\}$.

From now on, we let $p(z)$ be an analytic function in a neighborhood of the origin. Then we represent $p(z)$ by a power series $\sum_{n=0}^{\infty} p_{n} z^{n}$.

Theorem 1. Let $\gamma$ satisfy condition (H1). Then (11) has an analytic solution

$$
g(z)=\sum_{n=1}^{\infty} c_{n} z^{n}
$$

in a neighborhood of the origin such that $g(0)=0, g^{\prime}(0)=\eta$, where $\eta$ is a nonzero complex number.

Proof. Since $p(z)$ is analytic in a neighborhood of the origin, there exists a constant $\rho$ such that $\left|p_{n}\right| \leq \rho^{n-1}$ for $n \geq 1$. Substituting (13) into (12) and comparing coefficients of $z^{n}(n=1,2, \ldots)$, we get

$$
\begin{aligned}
\gamma c_{1} & =\gamma c_{1}, \\
2 \gamma^{2} c_{2} & =2 \gamma c_{2}-\gamma c_{1}^{2} p_{0}+2 c_{1}^{2} p_{2}
\end{aligned}
$$

and in general for $n \geq 1$

$$
\begin{aligned}
& (n+2)\left(\gamma^{n+2}-\gamma\right) c_{n+2}=\sum_{k=1}^{n} \sum_{i=1}^{k} \frac{i(n-k+1) \gamma^{2 k-i+2}}{k+1} \\
& \cdot c_{i} c_{k-i+1} c_{n-k+1}-\sum_{k=1}^{n+1} k p_{0} \gamma^{n-k+2} c_{k} c_{n-k+2} \\
& -\sum_{k=1}^{n} \sum_{i=1}^{k} \frac{i(n-k+1) \gamma^{k+1}}{k+1} \\
& \cdot c_{i} c_{n-k+1} \sum_{l_{1}+\cdots+l_{m}=k-i+1}^{m=1,2, \ldots, k-i+1} p_{m} c_{l_{1}} \cdots c_{l_{m}}+\sum_{k=1}^{n+1} 2 k p_{2} c_{k} c_{n-k+2} \\
& +\sum_{k=1}^{n} \sum_{i=1}^{k} \frac{i(n-k+1)}{k+1} \\
& \cdot c_{i} c_{n-k+1} \sum_{\substack{l_{1}+\cdots+l_{m}=k-i+1 \\
m=1,2, \ldots, k-i+1}} p_{m+2}(m+2)(m+1) c_{l_{1}} \cdots c_{l_{m}}
\end{aligned}
$$

The first expression allows us to choose $c_{1}=\eta \neq 0$ and the second expression implies $c_{2}=\left(2 p_{2}-\gamma p_{0}\right) c_{1}^{2} / 2 \gamma(\gamma-1)$. Consequently, the sequence $\left\{c_{n}\right\}_{n=3}^{\infty}$ is successively determined by the last expression in a unique manner. This implies that (11) has a formal power series solution.

Next, we show that the power series $g(z)$ converges in a neighborhood of the origin. Since $\left|p_{n}\right| \leq \rho^{n-1}$ and 
$\lim _{n \rightarrow \infty}\left(1 /\left(\gamma^{n+1}-1\right)\right)=-1$ for $0<|\gamma|<1$, there exists a positive constant $M$ such that

$$
\begin{aligned}
& \left|c_{n+2}\right| \leq M\left[\sum_{k=1}^{n} \sum_{i=1}^{k}\left|c_{i}\right|\left|c_{k-i+1}\right|\left|c_{n-k+1}\right|\right. \\
& +2 \sum_{k=1}^{n+1}\left|c_{k}\right|\left|c_{n-k+2}\right|+\sum_{k=1}^{n} \sum_{i=1}^{k}\left|c_{i}\right|\left|c_{n-k+1}\right| \\
& \sum_{\substack{l_{1}+\cdots+l_{m}=k-i+1 \\
m=1,2, \ldots, k-i+1}}\left|c_{l_{1}}\right| \cdots\left|c_{l_{m}}\right|+\sum_{k=1}^{n} \sum_{i=1}^{k}\left|c_{i}\right|\left|c_{n-k+1}\right| \\
& \left.\sum_{\substack{l_{1}+\cdots+l_{m}=k-i+1 \\
m=1,2, \ldots, k-i+1}}(m+2)(m+1)\left|c_{l_{1}}\right| \cdots\left|c_{l_{m}}\right|\right] .
\end{aligned}
$$

Let us define a power series $\sum_{n=1}^{\infty} a_{n} z^{n}$, where a positive sequence $\left\{a_{n}\right\}_{n=1}^{\infty}$ is determined by $a_{1}=|\eta|, a_{2}=\mid\left(2 p_{2}-\right.$ $\left.\gamma p_{0}\right) c_{1}^{2}|/| 2 \gamma(\gamma-1) \mid$ and for $n \geq 1$

$$
\begin{aligned}
& a_{n+2}=M\left[\sum_{k=1}^{n} \sum_{i=1}^{k} a_{i} a_{k-i+1} a_{n-k+1}+2 \sum_{k=1}^{n+1} a_{k} a_{n-k+2}\right. \\
& +\sum_{k=1}^{n} \sum_{i=1}^{k} a_{i} a_{n-k+1} \sum_{\substack{l_{1}+\cdots+l_{m}=k-i+1 \\
m=1,2, \ldots, k-i+1}} a_{l_{1}} \cdots a_{l_{m}} \\
& +\sum_{k=1}^{n} \sum_{\substack{i=1 \\
a_{i}}}^{k} a_{n-k+1} \\
& \quad \sum_{\substack{l_{1}+\cdots+l_{m}=k-i+1 \\
m=1,2, \ldots, k-i+1}}(m+2)(m+1) a_{l_{1}} \cdots a_{l_{m}}
\end{aligned}
$$

It follows that $\left|c_{n}\right| \leq a_{n}$ for $n \geq 1$. That is, $\sum_{n=1}^{\infty} a_{n} z^{n}$ is a majorant series of $\sum_{n=1}^{\infty} c_{n} z^{n}$. We show that $\sum_{n=1}^{\infty} a_{n} z^{n}$ is analytic in a neighborhood of the origin. Note that if we let $A(z)=\sum_{n=1}^{\infty} a_{n} z^{n}$, then

$$
\begin{aligned}
& A(z)= \sum_{n=1}^{\infty} a_{n} z^{n}=|\eta| z+a_{2} z^{2} \\
&+M\left[\sum_{n=1}^{\infty}\left(\sum_{k=1}^{n} \sum_{i=1}^{k} a_{i} a_{k-i+1} a_{n-k+1}\right) z^{n+2}\right.
\end{aligned}
$$

$$
\begin{aligned}
& +2 \sum_{n=1}^{\infty}\left(\sum_{k=1}^{n+1} a_{k} a_{n-k+2}\right) z^{n+2}+\sum_{n=1}^{\infty}\left(\sum_{k=1}^{n} \sum_{i=1}^{k} a_{i} a_{n-k+1}\right. \\
& \left.\sum_{\substack{l_{1}+\cdots+l_{m}=k-i+1 \\
m=1,2, \ldots, k-i+1}} a_{l_{1}} \cdots a_{l_{m}}\right) z^{n+2} \\
& +\sum_{n=1}^{\infty}\left(\sum_{k=1}^{n} \sum_{i=1}^{k} a_{i} a_{n-k+1}\right. \\
& \left.\left.\cdot \sum_{\substack{l_{1}+\cdots+l_{m}=k-i+1 \\
m=1,2, \ldots, k-i+1}}(m+2)(m+1) a_{l_{1}} \cdots a_{l_{m}}\right) z^{n+2}\right] \\
& =|\eta| z+\left|\frac{\left(2 p_{2}-\gamma p_{0}\right) c_{1}^{2}}{2 \gamma(\gamma-1)}\right| z^{2}+M\left[(A(z))^{3}\right. \\
& +2(A(z))^{2}-2|\eta|^{2} z^{2}+\frac{(A(z))^{3}}{1-A(z)} \\
& \left.+\frac{2(A(z))^{3}\left(3-3 A(z)+A(z)^{2}\right)}{(1-A(z))^{3}}\right] \text {. }
\end{aligned}
$$

Consider the equation

$$
\begin{aligned}
& T(z, A)=A-|\eta| z-\left|\frac{\left(2 p_{2}-\gamma p_{0}\right) c_{1}^{2}}{2 \gamma(\gamma-1)}\right| z^{2}-M\left[A^{3}\right. \\
& \left.\quad+2 A^{2}-2|\eta|^{2} z^{2}+\frac{A^{3}}{1-A}+\frac{2 A^{3}\left(3-3 A+A^{2}\right)}{(1-A)^{3}}\right] \\
& \quad=0 .
\end{aligned}
$$

Since $T$ is continuous in a neighborhood of the origin, $T(0,0)=0$, and $T_{A}^{\prime}(0,0)=1 \neq 0$, the implicit function theorem implies that there exists a unique function $A(z)$ which is analytic in a neighborhood of the origin with a positive radius. Because $A(z)$ is a majorant series of $g(z), g(z)$ is also analytic in a neighborhood of the origin with a positive radius. This completes the proof.

Now, we consider an analytic solution $g(z)$ of the auxiliary equation (11) in the case of $\gamma$ satisfies condition (H2). In order to study the existence of $g(z)$ under the Brjuno condition, we will recall the definition of Brjuno number and some basic facts. As stated in [10], for a real number $\theta$, we let $[\theta]$ be an integer part of $\theta$ and let $\{\theta\}=\theta-[\theta]$ be a fractional part of $\theta$. Observe that if $\theta$ is an irrational number, then it has a unique expression of Gauss's continued fraction

$$
\theta=d_{0}+\theta_{0}=d_{0}+\frac{1}{d_{1}+\theta_{1}}=\cdots,
$$


denoted simply by $\theta=\left[d_{0} ; d_{1}, \ldots, d_{n}, \ldots\right]$, where $d_{j}$ 's and $\theta_{j}$ 's are calculated by the following algorithm:

(a) $d_{0}=[\theta], \theta_{0}=\{\theta\}$;

(b) $d_{n}=\left[1 / \theta_{n-1}\right], \theta_{n}=\left\{1 / \theta_{n-1}\right\}$ for all $n \geq 1$.

Define the sequences $\left\{p_{n}\right\}_{n \in \mathbb{N}}$ and $\left\{q_{n}\right\}_{n \in \mathbb{N}}$ by the following recursive relation:

$$
\begin{aligned}
q_{-2} & =1, \\
q_{-1} & =0 \\
q_{n} & =d_{n} q_{n-1}+q_{n-2} ; \\
p_{-2} & =0 \\
p_{-1} & =1, \\
p_{n} & =d_{n} p_{n-1}+p_{n-2} .
\end{aligned}
$$

Note that $p_{n} / q_{n}=\left[d_{0} ; d_{1}, \ldots, d_{n}\right]$. For each $\theta \in \mathbb{R} \backslash \mathbb{Q}$, we consider an arithmetical function $B(\theta)=\sum_{n \geq 0}\left(\log q_{n+1} / q_{n}\right)$. When $\theta$ satisfies condition $B(\theta)<+\infty$, we call it a Brjuno number. Consider $\theta=\left[d_{0} ; d_{1}, \ldots, d_{n}, \ldots\right]$ in which for each $n \geq 0, d_{n+1} \leq c e^{d_{n}}$, where $c$ is a positive constant. We can show that $\theta$ is a Brjuno number but is not a Diophantine number. Therefore, Brjuno condition is weaker than the Diophantine condition. So, condition (H2) contains both Diophantine condition and a part of $\gamma$ near resonance.

Let $\theta \in \mathbb{R} \backslash \mathbb{Q}$ and $\left\{q_{n}\right\}_{n \in N}$ be the sequence of partial denominators of Gauss's continued fraction for $\theta$. As in [10], let

$$
\begin{aligned}
A_{k} & =\left\{n \geq 0:\|n \theta\| \leq \frac{1}{8 q_{k}}\right\}, \\
E_{k} & =\max \left\{q_{k}, \frac{q_{k+1}}{4}\right\}, \\
\eta_{k} & =\frac{q_{k}}{E_{k}} .
\end{aligned}
$$

Let $A_{k}^{*}$ be the set of integers $j \geq 0$ such that either $j \in A_{k}$ or for some $j_{1}$ and $j_{2}$ in $A_{k}$, with $j_{2}-j_{1}<E_{k}$, one has $j_{1}<j<j_{2}$ and $q_{k}$ divides $j-j_{1}$. For any nonnegative integer $n$, we define

$$
l_{k}(n)=\max \left\{\left(1+\eta_{k}\right) \frac{n}{q_{k}}-2,\left(m_{n} \eta_{k}+n\right) \frac{1}{q_{k}}-1\right\},
$$

where $m_{n}=\max \left\{j: 0 \leq j \leq n, j \in A_{k}^{*}\right\}$. Let $h_{k}: \mathbb{N} \rightarrow \mathbb{R}^{+}$ be a function defined by

$$
h_{k}(n)= \begin{cases}\frac{m_{n}+\eta_{k} n}{q_{k}}-1 & \text { if } m_{n}+q_{k} \in A_{k}^{*}, \\ l_{k}(n) & \text { if } m_{n}+q_{k} \notin A_{k}^{*} .\end{cases}
$$

Let $g_{k}(n):=\max \left\{h_{k}(n),\left[n / q_{k}\right]\right\}$, and let $k(n)$ be defined by condition $q_{k(n)} \leq n \leq q_{k(n)+1}$. Note that $k(n)$ is nondecreasing.

Lemma 2 (Davie's lemma [11]). Let

$$
K(n)=n \log 2+\sum_{k=0}^{k(n)} g_{k}(n) \log \left(2 q_{k+1}\right)
$$

Then

(a) there is a universal constant $\xi>0$ (independent of $n$ and $\theta$ ) such that

$$
K(n) \leq n(B(\theta)+\xi)
$$

(b) $K\left(n_{1}\right)+K\left(n_{2}\right) \leq K\left(n_{1}+n_{2}\right)$ for all $n_{1}$ and $n_{2}$,

(c) $-\log \left|\gamma^{n}-1\right| \leq K(n)-K(n-1)$.

Theorem 3. Assume that $\gamma$ satisfies condition (H2). Then there exists an analytic solution

$$
g(z)=\sum_{n=1}^{\infty} c_{n} z^{n}
$$

of (11) in a neighborhood of the origin such that $g(0)=0$, $g^{\prime}(0)=\eta$, where $\eta$ is a nonzero complex number.

Proof. We now imitate the proof of Theorem 1 with approximate new bound. The sequence $\left\{c_{n}\right\}_{n=1}^{\infty}$ is defined similar to the proof of Theorem 1 . Note that $c_{1}=\eta \neq 0$ and $c_{2}=$ $\left(2 p_{2}-\gamma p_{0}\right) c_{1}^{2} / 2 \gamma(\gamma-1)$. Since $|\gamma|=1$ and $p(z)$ is analytic near the origin, there exists a positive constant $N$ so that for $n \geq 1$

$$
\begin{aligned}
& \left|c_{n+2}\right| \leq \frac{N}{\left|\gamma^{n+1}-1\right|}\left[\sum_{k=1}^{n} \sum_{i=1}^{k}\left|c_{i}\right|\left|c_{k-i+1}\right|\left|c_{n-k+1}\right|\right. \\
& +2 \sum_{k=1}^{n+1}\left|c_{k}\right|\left|c_{n-k+2}\right|+\sum_{k=1}^{n} \sum_{i=1}^{k}\left|c_{i}\right|\left|c_{n-k+1}\right| \\
& \sum_{\substack{l_{1}+\cdots+l_{m}=k-i+1 \\
m=1,2, \ldots, k-i+1}}\left|c_{l_{1}}\right| \cdots\left|c_{l_{m}}\right|+\sum_{k=1}^{n} \sum_{i=1}^{k}\left|c_{i}\right|\left|c_{n-k+1}\right| \\
& \left.\sum_{\substack{l_{1}+\cdots+l_{m}=k-i+1 \\
m=1,2, \ldots, k-i+1}}(m+2)(m+1)\left|c_{l_{1}}\right| \cdots\left|c_{l_{m}}\right|\right] .
\end{aligned}
$$

To construct a governing series of $g(z)$, we let $\left\{B_{n}\right\}_{n=1}^{\infty}$ be a nonnegative sequence determined by $B_{1}=|\eta|, B_{2}=\mid\left(2 p_{2}-\right.$ $\left.\gamma p_{0}\right) \eta^{2}|/| 2 \gamma(\gamma-1) \mid$ and for all $n \geq 1$

$$
\begin{gathered}
B_{n+2}=N\left[\sum_{k=1}^{n} \sum_{i=1}^{k} B_{i} B_{k-i+1} B_{n-k+1}+2 \sum_{k=1}^{n+1} B_{k} B_{n-k+2}\right. \\
+\sum_{k=1}^{n} \sum_{i=1}^{k} B_{i} B_{n-k+1} \sum_{\substack{l_{1}+\cdots+l_{m}=k-i+1 \\
m=1,2, \ldots, k-i+1}} B_{l_{1}} \cdots B_{l_{m}}
\end{gathered}
$$




$$
\begin{aligned}
& +\sum_{k=1}^{n} \sum_{i=1}^{k} B_{i} B_{n-k+1} \\
& \left.\sum_{\substack{l_{1}+\cdots+l_{m}=k-i+1 \\
m=1,2, \ldots, k-i+1}}(m+2)(m+1) B_{l_{1}} \cdots B_{l_{m}}\right] .
\end{aligned}
$$

From this construction, we can demonstrate that a power series $B(z)=\sum_{n=1}^{\infty} B_{n} z^{n}$ satisfies the implicit functional equation

$$
\begin{aligned}
& R(z, B(z))=B(z)-|\eta| z-a_{2} z^{2}-N\left[(B(z))^{3}\right. \\
& +2(B(z))^{2}-2|\eta|^{2} z^{2}+\frac{(B(z))^{3}}{1-B(z)} \\
& \left.+\frac{2(B(z))^{3}\left(3-3 B(z)+B(z)^{2}\right)}{(1-B(z))^{3}}\right]=0
\end{aligned}
$$

with $R(0,0)=0$ and $R_{B}^{\prime}(0,0)=1 \neq 0$. This yields the power series $B(z)$ converges in a neighborhood of the origin. Hence, there exists a positive constant $T$ such that $B_{n} \leq T^{n}$ for $n \geq 1$.

Let $K$ be a function defined in Lemma 2. By mathematical induction, we can show that for $n \in \mathbb{N} \cup\{0\}$

$$
\left|c_{n+1}\right| \leq B_{n+1} e^{K(n)} .
$$

Lemma 2 yields $\lim _{n \rightarrow \infty}\left(\left|c_{n+1}\right|\right)^{1 / n} \leq T e^{B(\theta)+\xi}$. This implies that $g(z)$ has a convergence radius at least $\left(T e^{B(\theta)+\xi}\right)^{-1}$. The proof is completed.

Finally, we consider the case that $\gamma$ satisfies condition (H3). In this case, $\gamma$ is not only on the unit circle, but also a root of unity. Let $\left\{D_{n}\right\}_{n=1}^{\infty}$ be a sequence defined by $D_{1}=|\eta|$, $D_{2}=\Gamma A$ with $A=\left|\left(2 p_{2}-\gamma p_{0}\right) c_{1}^{2}\right| /|2 \gamma|, \Gamma=\max \{1 / \mid \gamma-$ $\left.1|, 1 /| \gamma^{2}-1|, \ldots, 1 /| \gamma^{p-1}-1 \mid\right\}$, and

$$
\begin{gathered}
D_{n+2}=\Gamma N\left[\sum_{k=1}^{n} \sum_{i=1}^{k} D_{i} D_{k-i+1} D_{n-k+1}+2 \sum_{k=1}^{n+1} D_{k} D_{n-k+2}\right. \\
+\sum_{k=1}^{n} \sum_{i=1}^{k} D_{i} D_{n-k+1} \sum_{\substack{l_{1}+\cdots+l_{m}=k-i+1 \\
m=1,2, \ldots, k-i+1}} D_{l_{1}} \cdots D_{l_{m}} \\
+\sum_{k=1}^{n} \sum_{\substack{i=1 \\
D_{i}}}^{k} D_{n-k+1} \\
\sum_{\substack{l_{1}+\cdots+l_{m}=k-i+1 \\
m=1,2, \ldots, k-i+1}}(m+2)(m+1) D_{l_{1}} \cdots D_{l_{m}}
\end{gathered}
$$

where $N$ is a positive constant defined as in the proof of Theorem 3.
Theorem 4. Assume that $\gamma$ satisfies condition (H3). Let $g(z)=$ $\sum_{n=1}^{\infty} c_{n} z^{n}$ be a power series determined by $c_{1}=\eta \neq 0, c_{2}=$ $\left(2 p_{2}-\gamma p_{0}\right) c_{1}^{2} / 2 \gamma$, and

$$
(n+2) \gamma\left(\gamma^{n+1}-1\right) c_{n+2}=\Theta(n, \gamma), \quad n=1,2, \ldots,
$$

where

$$
\begin{aligned}
& \Theta(n, \gamma)=\sum_{k=1}^{n} \sum_{i=1}^{k} \frac{i(n-k+1) \gamma^{2 k-i+2}}{k+1} \cdot c_{i} c_{k-i+1} c_{n-k+1} \\
& -\sum_{k=1}^{n+1} k c_{k} p_{0} \gamma^{n-k+2} c_{n-k+2}-\sum_{k=1}^{n} \sum_{i=1}^{k} \frac{i(n-k+1) \gamma^{k+1}}{k+1} \\
& \cdot c_{i} c_{n-k+1} \sum_{\substack{l_{1}+\cdots+l_{m}=k-i+1 \\
m=1,2, \ldots, k-i+1}} p_{m} c_{l_{1}} \cdots c_{l_{m}} \\
& +\sum_{k=1}^{n+1} 2 k p_{2} c_{k} c_{n-k+2}+\sum_{k=1}^{n} \sum_{i=1}^{k} \frac{i(n-k+1)}{k+1} \\
& \cdot c_{i} c_{n-k+1} \sum_{\substack{l_{1}+\cdots+l_{m}=k-i+1 \\
m=1,2, \ldots, k-i+1}} p_{m+2}(m+2)(m+1) \\
& \cdot c_{l_{1}} \cdots c_{l_{m}} \cdot{ }^{n}
\end{aligned}
$$

If $\Theta(v p-1, \gamma)=0$ for $v=1,2, \ldots$, then (11) has an analytic solution $g(z)$ in a neighborhood of the origin such that $g(0)=0, g^{\prime}(0)=\eta$, and $g^{(v p+1)}(0)=(v p+1) ! \eta_{v p+1}$, where $\eta_{v p+1}$ is an arbitrary constant satisfying $\left|\eta_{v p+1}\right| \leq D_{v p+1}$, where $\left\{D_{n}\right\}_{n=1}^{\infty}$ is defined as in (32).

Otherwise, if $\Theta(v p-1, \gamma) \neq 0$ for some $v=1,2, \ldots$, then (11) has no analytic solution in a neighborhood of the origin.

Proof. Observe that if $\eta=0$ then $g(z) \equiv 0$ is a trivial analytic solution of (11). So, we consider only the case $\eta \neq 0$.

If $\Theta(v p-1, \gamma) \neq 0$ for some positive number $v$, then $(v p+1) \gamma\left(\gamma^{v p}-1\right) c_{v p+1} \neq 0$. But $(H 3)$ implies $\gamma^{v p}-1=0$, which is a contradiction. This concludes that (11) has no analytic solution in a neighborhood of the origin.

Assume that $\Theta(v p-1, \gamma)=0$ for $v=1,2, \ldots$ Then $(v p+$ 1) $\gamma\left(\gamma^{v p}-1\right) c_{v p+1}=0$. So, there are infinitely many choices of $c_{v p+1}$. Choose $c_{v p+1}=\eta_{v p+1}$ so that $\left|\eta_{v p+1}\right| \leq D_{v p+1}$, where $D_{v p+1}$ is defined in (32).

Note that $\left|\gamma^{n+1}\right|^{-1} \leq \Gamma$ for $n \neq v p-1$, where $\Gamma=$ $\max \left\{1 /|\gamma-1|, 1 /\left|\gamma^{2}-1\right|, \ldots, 1 /\left|\gamma^{p-1}-1\right|\right\}$. We can see that

$$
\begin{gathered}
\left|c_{n+2}\right| \leq \Gamma N\left[\sum_{k=1}^{n} \sum_{i=1}^{k}\left|c_{i}\right|\left|c_{k-i+1}\right|\left|c_{n-k+1}\right|\right. \\
+2 \sum_{k=1}^{n+1}\left|c_{k}\right|\left|c_{n-k+2}\right|+\sum_{k=1}^{n} \sum_{i=1}^{k}\left|c_{i}\right|\left|c_{n-k+1}\right|
\end{gathered}
$$




$$
\begin{aligned}
& \sum_{\substack{l_{1}+\cdots+l_{m}=k-i+1 \\
m=1,2, \ldots, k-i+1}}\left|c_{l_{1}}\right| \cdots\left|c_{l_{m}}\right|+\sum_{k=1}^{n} \sum_{i=1}^{k}\left|c_{i}\right|\left|c_{n-k+1}\right| \\
& \left.\sum_{\substack{l_{1}+\cdots+l_{m}=k-i+1 \\
m=1,2, \ldots, k-i+1}}(m+2)(m+1)\left|c_{l_{1}}\right| \cdots\left|c_{l_{m}}\right|\right]
\end{aligned}
$$

for $n \neq v p-1, v=1,2, \ldots$.

Likewise, the remaining proof is similar to one of Theorem 1. Consider the implicit functional equation

$$
\begin{aligned}
& H(z, R)=R-|\eta| z-\frac{\left(2 p_{2}-\gamma p_{0}\right) c_{1}^{2}}{2 \gamma} \cdot z^{2}-N\left[R^{3}\right. \\
& \left.\quad+2 R^{2}-2|\eta|^{2} z^{2}+\frac{R^{3}}{1-R}+\frac{2 R^{3}\left(3-3 R+R^{2}\right)}{(1-R)^{3}}\right] \\
& \quad=0 .
\end{aligned}
$$

Since $H(0,0)=0, H_{R}^{\prime}(0,0)=1 \neq 0$, the implicit function theorem implies that there exists a unique function $R(z)$ which is analytic in a neighborhood of the origin with a positive radius. We can show that the power series $R(z)=$ $\sum_{n=1}^{\infty} D_{n} z^{n}$ in which $\left\{D_{n}\right\}_{n=1}^{\infty}$ is determined by (32) satisfies (36). Moreover, $\left|c_{n}\right| \leq D_{n}$ for $n \geq 1$. That is, $R(z)$ is a majorant series of $g(z)$. Then $g(z)$ converges in a neighborhood of the origin. This completes the proof.

Theorem 5. Let $g(z)=\sum_{n=1}^{\infty} c_{n} z^{n}$ be an analytic solution in a neighborhood of the origin of (11), with $g(0)=0, g^{\prime}(0)=$ $\eta \neq 0$, which is obtained from Theorem 1, Theorem 3, or Theorem 4. Then (10) has an analytic solution of the form $y(z)=g\left(\gamma g^{-1}(z)\right)$ in a neighborhood of the origin.

Proof. Since $g^{\prime}(0)=\eta \neq 0, g^{-1}(z)$ is analytic in a neighborhood of $g(0)=0$.

Let $y(z)=g\left(\gamma g^{-1}(z)\right)$. Then

$$
\begin{aligned}
y^{\prime \prime}(z)-p^{\prime \prime}(z) & =\frac{\gamma^{2} g^{\prime \prime}\left(\gamma g^{-1}(z)\right)}{\left(g^{\prime}\left(g^{-1}(z)\right)\right)^{2}}-\frac{\gamma g^{\prime}\left(\gamma g^{-1}(z)\right) g^{\prime \prime}\left(g^{-1}(z)\right)}{\left(g^{\prime}\left(g^{-1}(z)\right)\right)^{3}}-p^{\prime \prime}(z) \\
& =\frac{\gamma^{2} g^{\prime \prime}\left(\gamma g^{-1}(z)\right)\left(g^{\prime}\left(g^{-1}(z)\right)\right)-\gamma g^{\prime}\left(\gamma g^{-1}(z)\right) g^{\prime \prime}\left(g^{-1}(z)\right)-p^{\prime \prime}(z)\left(g^{\prime}\left(g^{-1}(z)\right)\right)^{3}}{\left(g^{\prime}\left(g^{-1}(z)\right)\right)^{3}} \\
& =\frac{\left[g\left(\gamma^{2} g^{-1}(z)\right)-p\left(g\left(\gamma g^{-1}(z)\right)\right)\right] \gamma g^{\prime}\left(\gamma g^{-1}(z)\right)\left(g^{\prime}\left(g^{-1}(z)\right)\right)^{3}}{} \\
& =\left[g\left(\gamma g^{-1} g \gamma g^{-1}(z)\right)-p\left(g\left(\gamma g^{-1}(z)\right)\right)\right] \cdot \frac{\gamma^{\prime}\left(\gamma g^{-1}(z)\right)}{g^{\prime}\left(\gamma g^{-1}(z)\right)}=[y(y(z))-p(y(z))] y^{\prime}(z) .
\end{aligned}
$$

That is, $y(z)=g\left(\gamma g^{-1}(z)\right)$ is an analytic solution of (10). The proof is completed.

\section{Analytic Solutions of (5) via (10)}

In this section, we construct an analytic solution of (5) from an analytic solution of (10). Assume that $x(z)$ is an analytic solution of the functional differential equation (5) in a neighborhood of the origin. Since $x(z)$ is analytic in a neighborhood of the origin, $x(z)$ can be represented by Taylor's series

$$
\begin{aligned}
x(z) & =\sum_{n=0}^{\infty} \frac{x^{(n)}(0)}{n !} \cdot z^{n} \\
& =x(0)+x^{\prime}(0) z+\frac{x^{\prime \prime}(0) z^{2}}{2 !}+\cdots .
\end{aligned}
$$

Let $p(z)=\sum_{n=0}^{\infty} p_{n} z^{n}$, where $p_{0}=\alpha$ and $p_{1}=\beta$ and $p_{n}=$ $p^{(n)}(0) / n$ ! for $n \geq 2$. Since $x^{\prime}(z)=(1 / b)(y(z)-p(z))$, we have $x^{\prime}(0)=-\alpha / b$. Since $x^{\prime \prime}(z)=(1 / b)\left(y^{\prime}(z)-p^{\prime}(z)\right)$ and $y^{\prime}(0)=\gamma$, it follows that $x^{\prime \prime}(0)=(1 / b)(\gamma-\beta)$. This implies that $(\gamma-\beta) / b=x^{\prime \prime}(0)=x\left(p(0)+b x^{\prime}(0)\right)=x(\alpha-b \cdot(\alpha / b))=$ $x(0)$.

Since $x^{\prime \prime \prime}(z)=x^{\prime}\left(p(z)+b x^{\prime}(z)\right) \cdot\left(p^{\prime}(z)+b x^{\prime \prime}(z)\right)$, we have $x^{\prime \prime \prime}(0)=-\alpha \gamma / b$.

By using mathematical induction, we can show that

$$
\begin{aligned}
& \left(x\left(p(z)+b x^{\prime}(z)\right)\right)^{(m)}=\sum_{i=1}^{m} p_{i m}\left(p^{\prime}(z)\right. \\
& \quad+b x^{\prime \prime}(z), p^{\prime \prime}(z)+b x^{\prime \prime \prime}(z), \ldots, p^{(m)}(z) \\
& \left.\quad+b x^{(m+1)}(z)\right) x^{(i)}\left(p(z)+b x^{\prime}(z)\right),
\end{aligned}
$$

for $m=1,2,3, \ldots$, where $p_{\text {im }}(1 \leq i \leq m)$ is a polynomial with nonnegative coefficients. 
Therefore, the explicit form of an analytic solution of our equation is

$$
\begin{gathered}
x(z)=\frac{\gamma-\beta}{b}+\left(\frac{-\alpha}{b}\right) \cdot z+\frac{1}{2 !} \cdot\left(\frac{\gamma-\beta}{b}\right) \cdot z^{2}+\frac{1}{3 !} \\
\cdot\left(-\frac{\alpha \gamma}{b}\right) z^{3}+\sum_{m=2}^{\infty} \frac{\Gamma_{m}}{(m+2) !} \cdot z^{m+2},
\end{gathered}
$$

where $\Gamma_{m}$ denotes $x^{(m+2)}(0)$.

\section{Polynomial Solutions of (5)}

In this section, we let $p(z)$ be a polynomial. Then, we investigate the polynomial solution of (5).

Theorem 6. For a polynomial $p(z)$, the equation

$$
x^{\prime \prime}(z)=x\left(p(z)+b x^{\prime}(z)\right)
$$

has a nontrivial polynomial solution if and only if $p(z)=p_{0}$ with $p_{0} \neq 0$ or $p(z)=p_{0}+p_{1} z$ with $p_{1} \neq 0$.

Proof.

Necessity. Assume that $x(z)=\sum_{k=0}^{n} x_{k} z^{k}$ is a nontrivial polynomial solution of (41). Let $p(z)=\sum_{k=0}^{m} p_{k} z^{k}$ with $p_{m} \neq 0$.

Observe that $x^{\prime \prime}(z)=0$ when $n=0$. This implies $x(z) \equiv 0$. From now on, we let $n \geq 1$.

We consider 3 cases.

Case $1(m=0)$. That is, $p(z)=p_{0} \neq 0$. Equation (41) becomes

$$
\begin{aligned}
2 x_{2} & +6 x_{3} z+\cdots+n(n-1) x_{n} z^{n-2} \\
& =x_{0}+x_{1}(q(z))+\cdots+x_{n}(q(z))^{n},
\end{aligned}
$$

where $q(z)=\left(p_{0}+b x_{1}\right)+2 b x_{2} z+\cdots+n b x_{n} z^{n-1}$.

If $n=1$, then (42) changes to $0=x_{0}+x_{1}\left(p_{0}+b x_{1}\right)$.

Next, we consider $n \geq 2$.

Comparing coefficient of $z^{n(n-1)}$ in (42), we have $x_{n}=0$.

Equation (42) is reduced to

$$
\begin{aligned}
2 x_{2} & +\cdots+(n-1)(n-2) x_{n-1} z^{n-3} \\
& =x_{0}+x_{1}(q(z))+\cdots+x_{n-1}(q(z))^{n-1},
\end{aligned}
$$

where $q(z)=\left(p_{0}+b x_{1}\right)+2 b x_{2} z+\cdots+(n-1) b x_{n-1} z^{n-2}$.

Comparing coefficient of $z^{(n-1)(n-2)}$ in (43), we have $x_{n-1}=0$. Then, repeating the above method, we obtain $x_{n-2}=$ $\cdots=x_{2}=0$ and also have $0=x_{0}+x_{1}\left(p_{0}+b x_{1}\right)$.

By choosing an arbitrary nonzero $x_{1}$, say $\eta$, both situations yield a nontrivial solution $x(z)=-\eta\left(p_{0}+b \eta\right)+\eta z$.

Case $2(m=1)$. That is, $p(z)=p_{0}+p_{1} z$, where $p_{1} \neq 0$.

Equation (41) becomes

$$
\begin{aligned}
2 x_{2} & +6 x_{3} z+\cdots+n(n-1) x_{n} z^{n-2} \\
& =x_{0}+x_{1}(q(z))+\cdots+x_{n}(q(z))^{n},
\end{aligned}
$$

where $q(z)=\left(p_{0}+b x_{1}\right)+\left(p_{1}+2 b x_{2}\right) z+3 b x_{3} z^{2}+\cdots+n b x_{n} z^{n-1}$.
By comparing coefficient of constant term and $z$ in (44), we obtain $x(z) \equiv 0$ for $n=1$.

Next, we consider $n \geq 2$.

Comparing coefficient of $z^{n(n-1)}$ in (44), we have $x_{n}\left(n b x_{n}\right)^{n}=0$, which implies $x_{n}=0$. Therefore, (44) is reduced to

$$
\begin{aligned}
2 x_{2} & +6 x_{3} z+\cdots+(n-1)(n-2) x_{n-1} z^{n-3} \\
& =x_{0}+x_{1}(q(z))+\cdots+x_{n-1}(q(z))^{n-1},
\end{aligned}
$$

where $q(z)=\left(p_{0}+b x_{1}\right)+\left(p_{1}+2 b x_{2}\right) z+3 b x_{3} z^{2}+\cdots+$ $(n-1) b x_{n-1} z^{n-2}$.

Comparing coefficient of $z^{(n-1)(n-2)}$ in (45), we have $x_{n-1}=0$. Continuing this process, we obtain $2 x_{2}=x_{0}+$ $x_{1}(q(z))+x_{2}(q(z))^{2}$, where $q(z)=\left(p_{0}+b x_{1}\right)+\left(p_{1}+2 b x_{2}\right) z$. By comparing coefficient of $z^{2}$ together with $x_{2} \neq 0$, we obtain a nontrivial solution $x(z)=\left(-p_{1} / b\right)-\eta\left(p_{0}+b \eta\right)+\left(p_{1} / 2 b\right)\left(p_{0}+\right.$ $b \eta)^{2}+\eta z-\left(p_{1} / 2 b\right) z^{2}$, where $\eta$ is an arbitrary constant. Note that if $x_{2}=0$ then $x(z) \equiv 0$.

Case $3(m \geq 2)$. We consider 2 subcases.

Subcase $3.1(m<n-1)$. Equation (41) becomes

$$
\begin{aligned}
2 x_{2} & +6 x_{3} z+\cdots+n(n-1) x_{n} z^{n-2} \\
& =x_{0}+x_{1}(q(z))+\cdots+x_{n}(q(z))^{n},
\end{aligned}
$$

where $q(z)=\left(p_{0}+b x_{1}\right)+\cdots+\left(p_{m}+(m+1) b x_{m+1}\right) z^{m}+$ $(m+2) b x_{m+2} z^{m+1}+\cdots+n b x_{n} z^{n-1}$. By using method of undetermined coefficient, we obtain $x_{n}=\cdots=x_{m+2}=0$. Consequently, (46) is reduced to

$$
\begin{aligned}
2 x_{2} & +6 x_{3} z+\cdots+(m+1)(m) x_{m+1} z^{m-1} \\
& =x_{0}+x_{1}(q(z))+\cdots+x_{m+1}(q(z))^{m+1},
\end{aligned}
$$

where $q(z)=\left(p_{0}+b x_{1}\right)+\cdots+\left(p_{m}+(m+1) b x_{m+1}\right) z^{m}$.

Comparing coefficient of $z^{m(m+1)}$ in (47), we have $x_{m+1}\left(p_{m}+(m+1) b x_{m+1}\right)^{m+1}=0$. If $x_{m+1}=0$, then $x_{m}=\cdots=$ $x_{0}=0$. That is, $x(z) \equiv 0$ which is a contradiction. Therefore, $p_{m}+(m+1) b x_{m+1}=0$. Substituting this relation in (46) and repeating this process, we get $x_{m-k}=-p_{m-(k+1)} /(m-k) b$ for $k=-1, \ldots, m-2$. Using this fact in (47) and then comparing the coefficient of $z^{i}(i=1, \ldots, m-1)$, we obtain $x_{3}=\cdots=$ $x_{m+1}=0$. This yields $p_{m}=0$, which is a contradiction.

Subcase $3.2(m \geq n-1)$. Equation (41) becomes

$$
\begin{aligned}
2 x_{2} & +6 x_{3} z+\cdots+n(n-1) x_{n} z^{n-2} \\
& =x_{0}+x_{1}(q(z))+\cdots+x_{n}(q(z))^{n},
\end{aligned}
$$

where $q(z)=\left(p_{0}+b x_{1}\right)+\cdots+\left(p_{n-1}+n b x_{n}\right) z^{n-1}+p_{n} z^{n}+$ $\cdots+p_{m} z^{m}$. Comparing coefficient of $z^{n m}$ in (48) together with $p_{m} \neq 0$, we have $x_{n}=0$. Then, repeating the above method, we obtain $x(z) \equiv 0$ which is a contradiction. 
Thus, (41) has a nontrivial polynomial solution when $p(z)=p_{0}$ with $p_{0} \neq 0$ or $p(z)=p_{0}+p_{1} z$ with $p_{1} \neq 0$.

Sufficiency. It follows from the proof of Cases 1 and 2 in necessity part.

\section{Conflict of Interests}

The authors declare that there is no conflict of interests regarding the publication of this paper.

\section{Acknowledgments}

The first author is supported in part by Development and Promotion of Science and Talents Project (DPST). The second author is supported by National Research Council of Thailand and Khon Kaen University, Thailand (Grant no. kku fmis(580010)).

\section{References}

[1] R. Bellman and K. L. Cooke, Differential-Difference Equations, Academic Press, New York, NY, USA, 1963.

[2] J. Hale, Theory of Functional Differential Equations, Springer, New York, NY, USA, 2nd edition, 1977.

[3] E. Eder, "The functional-differential equation $x^{\prime}(t)=x(x(t))$," Journal of Differential Equations, vol. 54, no. 3, pp. 390-400, 1984.

[4] J.-G. Si and S. S. Cheng, "Analytic solutions of a functionaldifferential equation with state dependent argument," Taiwanese Journal of Mathematics, vol. 1, no. 4, pp. 471-480, 1997.

[5] F. Qiu and H. Z. Liu, "Analytic solutions of a first order iterative differential equation $x^{\prime}(z)=x(p(z)+b x(z))$," Demonstratio Mathematica, vol. 39, no. 1, pp. 81-90, 2006.

[6] P. Zhang, "Analytic solutions of a first order functional differential equation with a state derivative dependent delay," Electronic Journal of Differential Equations, vol. 2009, no. 51, pp. 1-8, 2009.

[7] J. G. Si and X. P. Wang, "Analytic solutions of a secondorder functional differential equation with a state derivative dependent delay," Results in Mathematics, vol. 39, no. 3-4, pp. 345-352, 2001.

[8] T. B. Liu and H. Li, "Analytic solutions of an iterative functional differential equation near resonance," International Journal of Differential Equations, vol. 2009, Article ID 145213, 14 pages, 2009.

[9] J.-G. Si and X.-P. Wang, "Analytic solutions of a secondorder functional-differential equation with a state derivative dependent delay," Colloquium Mathematicum, vol. 79, no. 2, pp. 273-281, 1999.

[10] Edgar Arturo Saenz Maldonado, Brjuno numbers and complex dynamics [M.S. thesis], Virginia Polytechnic Institute and State University, 2008.

[11] A. M. Davie, "The critical function for the semistandard map," Nonlinearity, vol. 7, no. 1, pp. 219-229, 1994. 


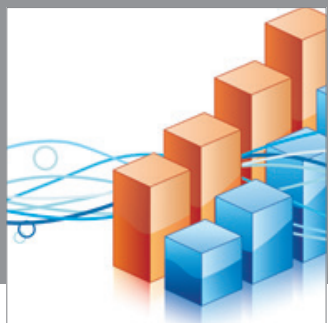

Advances in

Operations Research

mansans

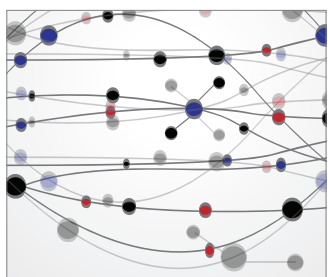

The Scientific World Journal
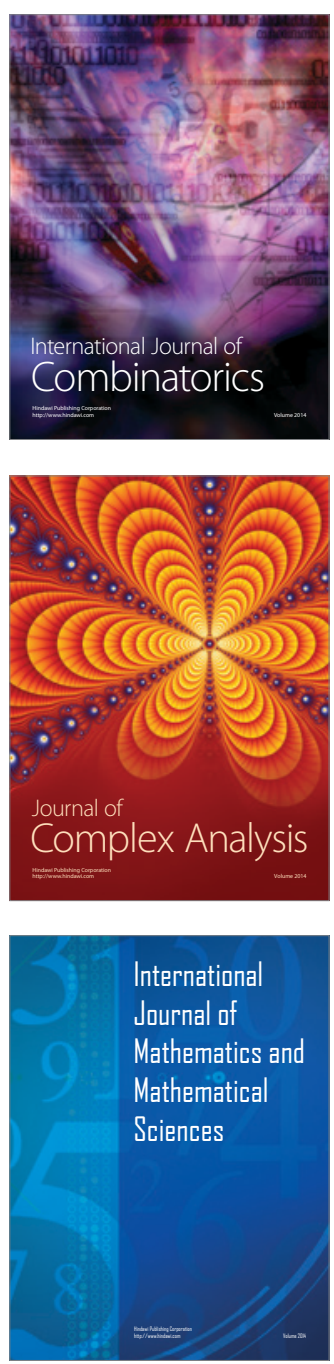
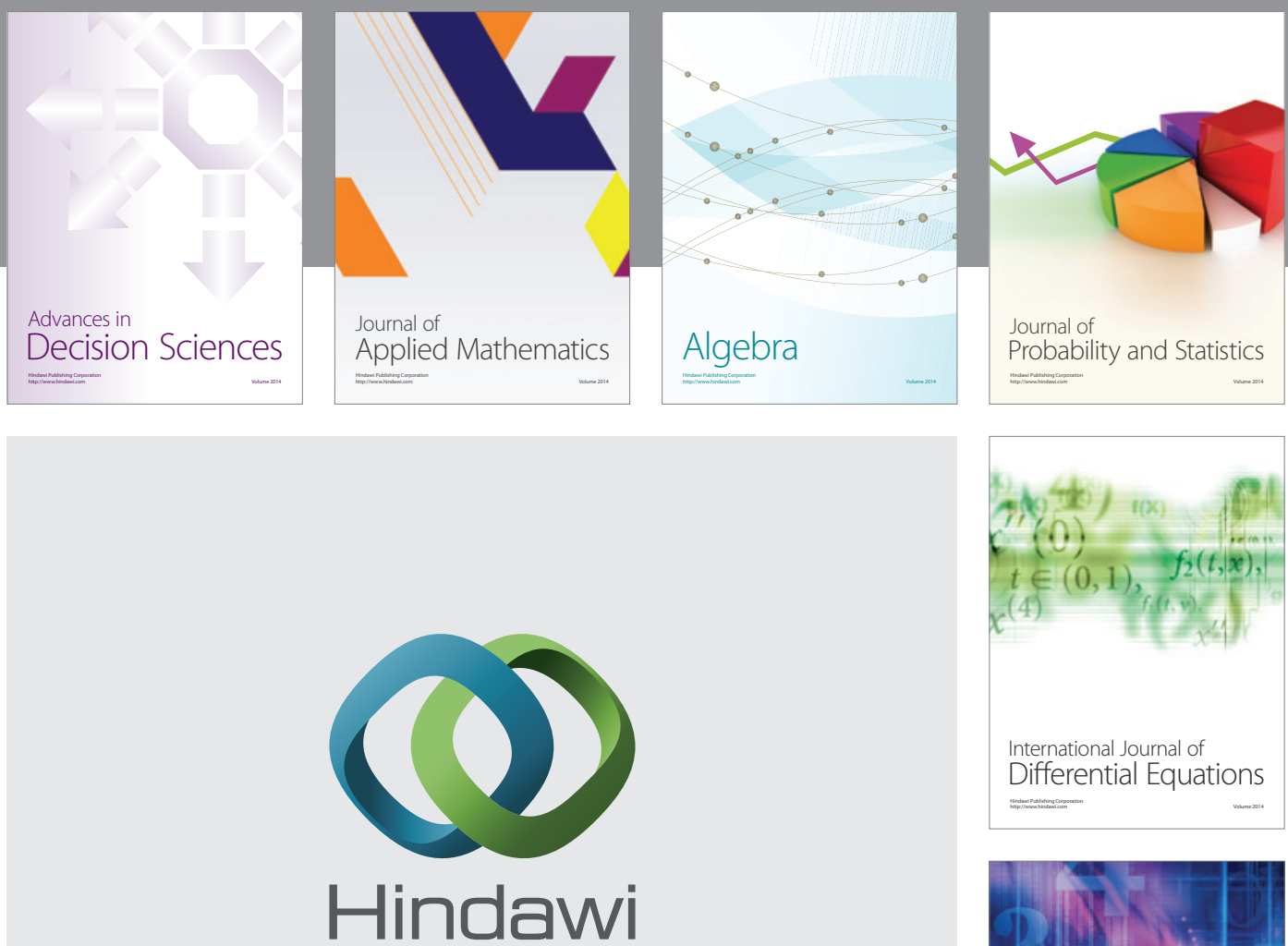

Submit your manuscripts at http://www.hindawi.com
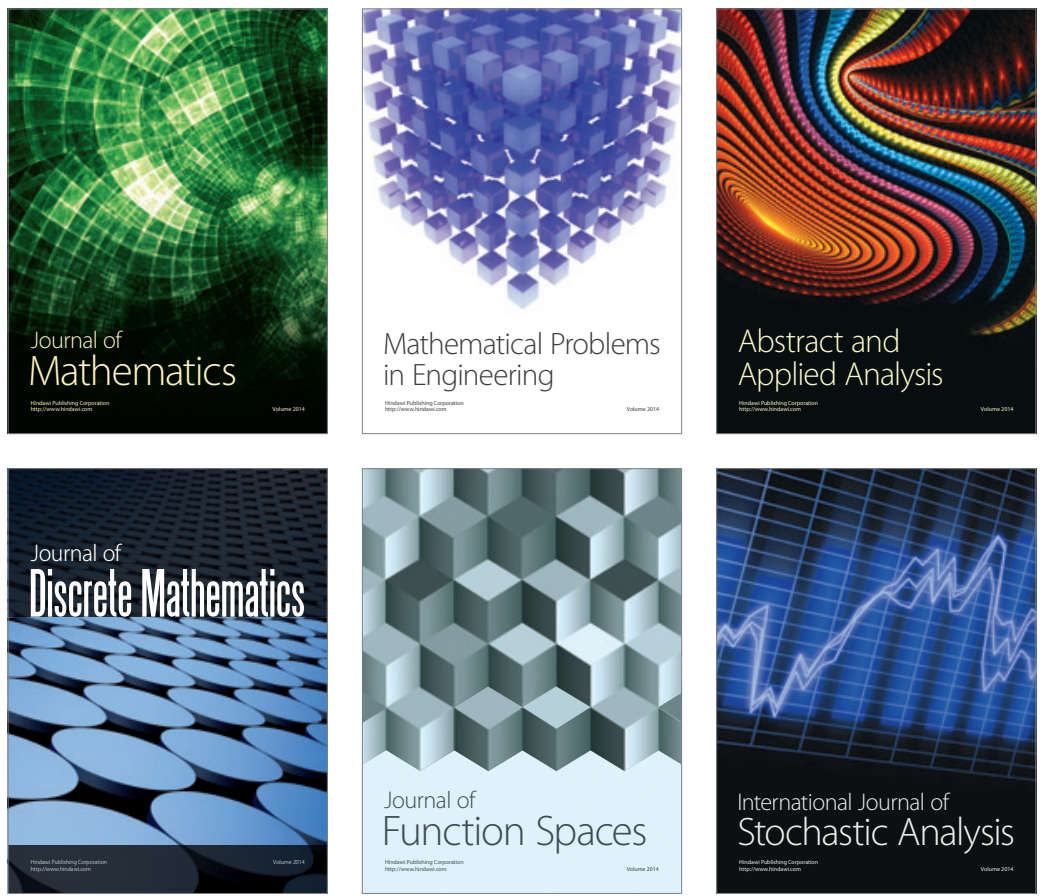

Journal of

Function Spaces

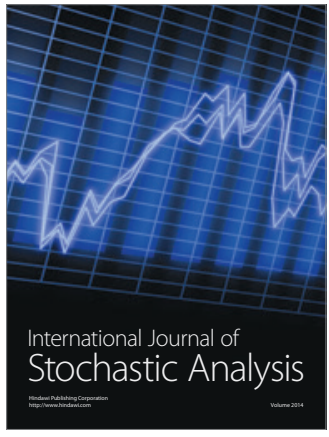

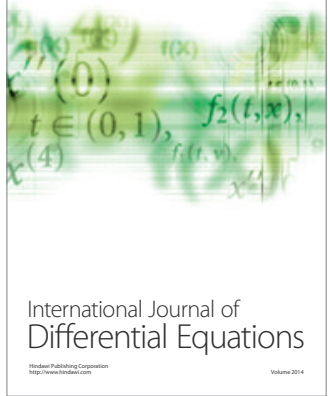
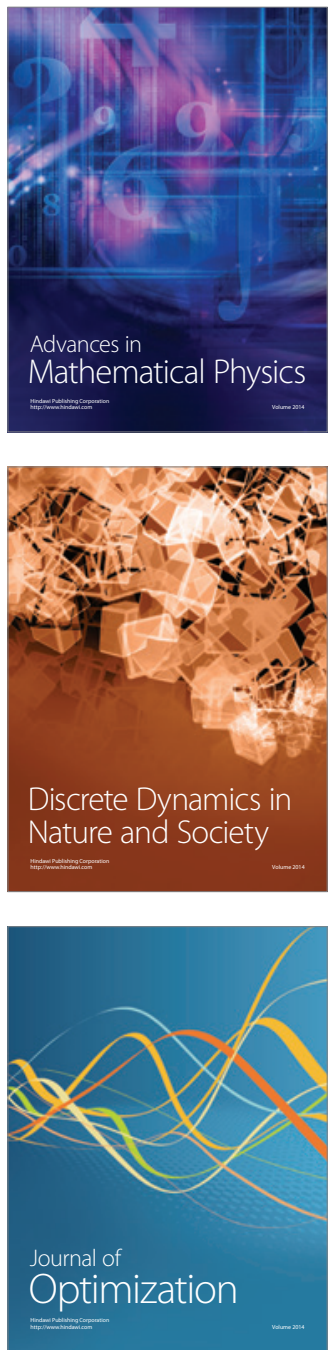